\title{
Relação do índice tornozelo-braquial como preditor de risco cardiovascular em pacientes submetidos a cateterismo cardíaco de uma cidade de Minas Gerais
}

\author{
Ankle-brachial index as a predictor of cardiovascular risk in patients undergoing cardiac \\ catheterization in a city in Minas Gerais
}
Relación del índice tobillo-brazo como predictor de riesgo cardiovascular en pacientes sometidos a cateterismo cardíaco en una ciudad de Minas Gerais

Gustavo Quintão Santana1, Isabela Freire Lage Reis ${ }^{1}$, Luiz Fernando Giuli Reis ${ }^{1}$, Maria Júlia Andrade Rodrigues $^{1 *}$, Newton Luiz Lombardi Fonseca Silveira1, Tânia Maria Gonçalves Quintão.

\section{RESUMO}

Objetivo: Analisar a prevalência de doença arterial obstrutiva periférica (DAOP), através do cálculo do índice tornozelo-braquial (ITB), e sua relação como preditor de risco cardiovascular em pacientes submetidos a cateterismo cardíaco. Métodos: Em um estudo transversal, com uma amostra de 250 pacientes submetidos a cateterismo cardíaco há, no máximo, 1 ano, foi realizada a medida do ITB de forma padronizada, utilizando-se umDoppler Vascular Portátil DV610 e um esfigmomanômetro, e foi elaborado um formulário de avaliação clínica, que foi aplicado para investigação de fatores de risco cardiovascular. Os dados obtidos foram transcritos em planilha digital e processados em software estatístico STATA v. 9.2. O estudo foi aprovado por Comitê de Ética em Pesquisa. Resultados: No presente estudo, a maior prevalência foi no sexo masculino (56\%) com média de idade de 63,5 $\pm 9,9$ anos. Dentro da amostra $63,3 \%$ apresentaram ITB normal e 36,4\% ITB alterado. A relação da alteração desse índice com etnia, etilismo, dislipidemia e idade apresentou-se estatisticamente significante $(p<0.05)$. Conclusão: $O$ cálculo de ITB se mostra eficaz e necessário na prática clínica como um preditor de risco importante para doença coronariana.

Palavras-chave: Índice tornozelo-braquial, Doença arterial periférica, Cateterismo cardíaco, Risco cardiovascular.

\begin{abstract}
Objective: To analyze the prevalence of peripheral arterial disease (PAD), through the calculation of the ankle-brachial index $(\mathrm{ABI})$, and its relationship as a risk predictor in patients undergoing cardiac catheterization. Methods: In a cross-sectional study, with a sample of 250 patients undergoing cardiac catheterization for at most 1 year, the measurement of the $A B I$ was performed in a standardized way, using a Portable Vascular Doppler DV610, a sphygmomanometer and a clinical evaluation form, which was applied to investigate cardiovascular risk factors. The data obtained were transcribed into a digital spreadsheet and processed in STATA v statistical software. 9.2. The study was approved by the Research Ethics Committee. Results: In the present study, the highest prevalence was of males $(56 \%)$ and the mean age was $63.5 \pm 9.9$ years. Within the sample, $63.3 \%$ had normal $\mathrm{ABI}$ and $36.4 \%$ had altered $\mathrm{ABI}$. The relationship between the altered index and ethnicity, alcoholism, dyslipidemia and age were statistically significant $(p<0.05)$.
\end{abstract}

\footnotetext{
${ }^{1}$ Faculdade de Medicina de Barbacena - Fundação José Bonifácio Lafayette de Andrada, Barbacena - MG.

*E-mail: majuandrade1999@gmail.com
}

SUBMETIDO EM: 10/2021 
Conclusion: The calculation of $\mathrm{ABI}$ is effective and necessary in clinical practice as a coronary artery disease risk predictor.

Keywords: Ankle brachial index, Peripheral arterial disease, Cardiac catheterization, Heart disease risk factors.

\section{RESUMEN}

Objetivo: Analizar la prevalencia de enfermidad arterial periférica (EAP), mediante el cálculo del índice tobillo-braquial (ITB), y su relación como predictor de riesgo cardiovascular en pacientes sometidos a cateterismo cardiaco. Métodos: Em um studio transversal, conducido com 250 pacientes sometidos a cateterismo cardíaco em el ultimo año, se realizo la medicíon estandarizada del ITB, utilizando Doppler Vascular Portátil DV610, esfigmomanómetro y se elaboro um formulario de evaluación clínica, el cual se aplicó para investigar factores de riesgo cardiovascular. Los datos obtenidos fueron transcritos a una hoja de cálculo digital e fueron procesados em el software estadístico STATA v. 9.2. El estúdio fue aprobado por el Comité de Ética em Investigación. Resultados: Em el presente studio, la mayor prevalência fue em hombres (56\%) com una edad promedio de 63,5 \pm 9,9 años. Em la muestra, 63,3\% tenía ITB normal y $36.4 \%$ ITB alterado. La relación del cambio del índice com etnia, alcoholism, dislipidemia y edad fue estadísticamente significativa $(p<0,05)$. Conclusión: El ITB es efectivo y necessario em la práctica clínica como un importante predictor de riesgo para la enfermidad de la artéria coronaria.

Palabras clave: Índicetobillo-braquial, Enfermedad arterial periférica, Cateterismo cardíaco, Riesgo de enfermedad cardiaca.

\section{INTRODUÇÃO}

A Doença Arterial Obstrutiva Periférica (DAOP) consiste em uma diminuição da perfusão tecidual, que surge em virtude de uma obstrução nos vasos sanguíneos arteriais dos membros superiores e dos membros inferiores, sendo maior a prevalência na extremidade inferior (KULLO IJ e ROOKE TW, 2016). A causa dessa enfermidade origina-se de fenômenos ateroscleróticos e aterotrombóticos, além de ser influenciada por aneurismas, arterites e êmbolos (OLIN JW, et al., 2016).

Trata-se de um processo crônico degenerativo obstrutivo aterosclerótico, que acomete os territórios periféricos, e tem uma prevalência estimada em $15 \%$ a $20 \%$ da população com mais de cinquenta e cinco anos, sendo que, nesses, $70 \%$ encontram-se assintomáticos por um longo tempo (NUNES FGF, et al. 2012). Seu diagnóstico baseia-se na investigação de sinais clínicos sugestivos, tais como a ausência de pulsos periféricos, a presença de frêmitos arteriais e as alterações de pele no membro afetado (PRESTI C, et al., 2015).

Estima- se uma intensa relação entre a DAOP e a Doença Arterial Coronariana (DAC) obstrutiva, daí a importância de sua detecção precoce. Observa-se que ambas são frequentemente encontradas em um mesmo paciente estando, portanto, associadas ao alto risco de eventos cardiovasculares como infarto agudo do miocárdio (IAM) (SABEDOTTI M, et al., 2014; LIU L, et al., 2020).

Os principais fatores de risco para aterosclerose na DAOP já estão muito bem documentados e se assemelham aos fatores de risco da DAC, como a idade avançada, considerando o avanço como maior probabilidade de desenvolver a doença, o sexo, em que homens estão mais susceptíveis, a diabetes mellitus tipo 2 (DM2), fator independe dos outros existentes na qual falhas no controle glicêmico e a duração da doença se traduzem em piora do quadro, a dislipidemia, como preditor de obstrução de vasos, o tabagismo, como o principal fator predisponente e a hipertensão arterial sistêmica (HAS), relacionado pela ausência de acompanhamento dos níveis pressóricos e pela extensão da enfermidade com o acometimento arterial (OLIVEIRA GHR, et al., 2011; ALVIM RO, et al., 2018). 
Os sintomas da DAOP, como a claudicação intermitente, podem se manifestar a partir da prática de exercícios físicos, quando se necessita de maior fluxo sanguíneo para os tecidos que estão exercendo a força, mas os vasos arteriais não são capazes de suprir a necessidade, ou até mesmo em repouso. Tal sintomatologia é decorrente da isquemia provocada pelas placas ateroscleróticas e consequente diminuição do calibre da artéria com aumento da resistência vascular. Devido a quantidade exorbitante de pacientes que possuem um quadro clínico ausente ou escasso para a realização do diagnóstico, apenas a anamnese completa e o exame físico tradicional podem não ser suficientes para identificar a doença (NEYELOFF JL, 2012; SABEDOTTI M, et al., 2014).

Estudos realizados, como o PARTNES, analisaram exames de rastreio e foi considerada a avaliação do Índice Torozelo-Braquial (ITB) como eficiente método no complemento da investigação. A DAOP é um potencial preditor de doenças cardiovasculares e há uma grande associação entre a alteração do ITB na presença dessa enfermidade e o seu potencial de evoluir com DAC (NEYELOFF JL, 2012; SABEDOTTI M, et al., 2014).

O ITB é um método de fácil realização, simples e não invasivo que possui baixo custo e apresenta elevada sensibilidade $(79 \%$ - 95\%) e alta especificidade (95\% - 100\%), sendo assim considerado um método altamente eficaz, possibilitando a detecção precoce da DAOP na população, incluindo os sintomáticos e os assintomáticos. Seu valor é obtido por meio da comparação entre a pressão arterial sistólica dos membros superiores e inferiores, dividindo um pelo outro, sendo que os parâmetros de normalidade estão entre 0,9 e 1,3 (FILHO JCJP, et al., 2015).

Para compreender o ITB, é necessário ressaltar que o esqueleto apendicular do corpo humano é simétrico e, se estiver sem alterações patológicas, possui parâmetros de normalidade bem semelhantes entre os membros. Dessa forma, os níveis pressóricos, em condições normais, deveriam ser próximos nos quatro membros, fazendo com que a razão da pressão dos superiores e dos inferiores ficasse em torno de 1. Assim, estudos nacionais constataram que um valor seguro para definir que o paciente tenha calcificação da camada média arterial é de ITB a partir de 1,3 e estudos internacionais definiram maior risco cardiovascular com ITB acima de 1,4, sendo que o maior fator de risco está associado ao ITB abaixo de 0,9 (ARAÚJO ALGS, et al., 2016)

Tal método é utilizado para detecção precoce de DAOP e denuncia um acometimento aterosclerótico em outros sítios, especialmente nas artérias coronárias (NUNES FGF, et al., 2012). Sendo assim, o objetivo do estudo foi avaliar os pacientes submetidos a angiografia coronariana através do ITB, permitindo assim, a detecção da prevalência de testes alterados em pacientes com DAC, mostrando a importância de seu uso no diagnóstico e na intervenção precoce das doenças arteriais, nas possíveis patologias cardiovasculares associadas e na sua gravidade (PETRACCO AM, et al., 2018).

A alta prevalência de doenças cardiovasculares, principalmente na população acima de 55 anos, mostra a grande importância de se pesquisar doença arterial obstrutiva periférica nos pacientes, uma vez que essa pode ser preditora de doença coronariana, aparecendo, muitas vezes, em pacientes ainda assintomáticos. Com a análise da literatura, foi possível estabelecer e comparar a associação de risco entre DAOP e DAC, uma vez que tal patologia é precedente de diversas comorbidades, que podem levar o paciente a estados graves e ao óbito (NUNES FGF, et al., 2012; SABEDOTTI M, et al., 2014; LIU L, et al., 2020).

O presente trabalho tem como objetivo analisar a prevalência de doença arterial obstrutiva periférica através do cálculo do índice tornozelo-braquial e sua relação como preditor de risco em pacientes submetidos ao cateterismo cardíaco.

\section{MÉTODOS}

Foi feito um estudo do tipo observacional transversal com uma amostra por conveniência formada por 250 pacientes, visando avaliar o ITB. Fizeram parte da amostra pacientes dos sexos feminino e masculino que foram submetidos a cateterismo cardíaco, há, no máximo, um ano, atendidos em hospitais e consultórios privados de uma cidade de Minas Gerais. Os pacientes foram esclarecidos e convidados a 
participar da pesquisa, na qual foram incluídos apenas aqueles que concordaram voluntariamente e assinaram o Termo de Consentimento Livre e Esclarecido (TCLE). Foram excluídos aqueles pacientes com amputação de membros superiores e membros superiores que impedisse a medida do ITB e aqueles com doença valvar grave.

Foi aplicado um formulário de avaliação clínica, construído com base nos escores de risco cardiovasculares de Framinghan, elucidando os fatores de risco nos pacientes elegíveis, como idade, sexo, etnia, tabagismo, etilismo, HAS, Índice de Massa Corporal (IMC), diabetes, prática de exercícios físicos, dislipidemia e histórico familiar para doenças cardiovasculares.

Além disso, foi realizada a medida do ITB, de forma padronizada e de acordo com o protocolo prescrito na literatura a partir da aferição da pressão arterial (PA) dos pacientes em decúbito dorsal, previamente em repouso por pelo menos 30 minutos. A PA foi aferida em ambos Membros Superiores (MMSS) e Membros Inferiores (MMII), com a utilização de um esfigmomanômetro e do Doppler braquial portátil DV610. Para o cálculo do ITB, foi utilizada a Pressão Arterial Sistólica (PAS) de ambos MMSS e MMII.

Assim, o cálculo final foi feito a partir do maior valor de PAS encontrado no membro inferior dividido pelo maior valor de PAS encontrado no membro superior, através da fórmula ITB= PAS Membro inferior/PAS Membro superior. Todos os dados coletados foram anotados adjacentes ao formulário aplicado e, posteriormente, transferidos a uma tabela para comparação e análise. Foram considerados os valores alterados de ITB aqueles $\leq 0,9$ ou $>1,3$, e os valores de referência para ITB normal foram de 0,91 a 1,30de acordo com diretrizes destinadas a prática clínica (FREITAS D, 2012; SOCIEDADE BRASILEIRA DE CARDIOLOGIA, 2019).

A amostra constituída por 250 pacientes define um IC95\% $\$ 6,2 \%$ para uma prevalência encontrada de $50 \%$ e $\pm 2,7 \%$ para uma prevalência encontrada de $5 \%$. A coleta de dados, através dos questionários, foi transcrita em planilha digital e processada em software estatístico STATA v. 9.2.

Através dos valores da coleta foram produzidas tabelas de frequências relativa e absoluta das variáveis de estudo. Além disso, foram calculadas as medidas de tendência central, posição e dispersão das variáveis quantitativas e foi calculado também o IC95\% das prevalências encontradas.

A associação existente entre as variáveis de estudo foi medida por testes de Quiquadro, Exato de Fisher, teste $t$ e teste de Mann-Whitney. As diferenças encontradas com valor $P \leq 0,05$ foram consideradas significativas.

Em relação aos aspectos éticos, o presente estudo foi submetido a Plataforma Brasil para avaliação e aprovação pelo Comitê de Ética em Pesquisa, sob o parecer 4.140.724 (CAAE: 34107020.2.0000.8307), de acordo com a Resolução 466/12 do Conselho Nacional de Saúde (CNS).

\section{RESULTADOS}

No presente estudo, a maior prevalência foi de indivíduos do sexo masculino (56\%) e de faiodermas $(45,2 \%)$. Entre a amostra populacional, o grupo que apresentou o ITB normal foi de $63,3 \%$ e o que apresentou o ITB alterado foi de $36,4 \%$.

$\mathrm{Na}$ coleta de dados, quando foi avaliado o tabagismo, a população representada foi de $22,8 \%$. Os extabagistas (abstinência de no mínimo 6 meses, sem recaídas) representaram 20,8\% da amostra e os não tabagistas (os que nunca fumaram) constituíam a maioria da população $(56,4 \%)$.

Houve presença significativa de sedentarismo, sendo $60 \%$ da amostra. A avaliação com relação aos portadores de HAS evidenciou a presença desse fator de risco em $91,6 \%$ da população amostral e o histórico familiar positivo para doença cardiovascular foi mostrado em $72,4 \%$ dos pacientes.

Quando foi avaliado o etilismo, a amostra foi representada por $18,8 \%$ de etilistas, os ex-etilistas (abstinência de no mínimo 6 meses, sem recaídas) representaram 11,6\%, e aqueles não etilistas (que nunca consumiram bebidas alcoólicas) foram representados por $69,6 \%$ da amostra, sendo a maior parcela do grupo estudado. 
Já os portadores de DM2 constituíram $35,6 \%$ da amostra, e aqueles com dislipidemia representaram $62,4 \%$ do grupo avaliado. A partir dos dados apresentados é possível analisar os fatores de risco dos pacientes em associação com os valores de ITB encontrados considerados normais (entre 0,91 e 1,30) e anormais $(\leq 0,9$ ou $>1,3)$ (Tabelas 1 e 2 ).

Tabela 1 - Fatores de risco cardiovasculares inerentes ao paciente associados ao ITB em pacientes submetidos a cateterismo de uma cidade de Minas Gerais em 2020

\begin{tabular}{lccc}
\hline Fator de risco & ITB normal $\mathbf{n}(\%)$ & ITB anormal $^{*} \mathbf{n}(\%)$ & Total $\mathbf{n}(\%)$ \\
\hline Leucoderma & $76(71,7)$ & $30(28,3)$ & $106(42,4)$ \\
Faioderma & $64(56,6)$ & $49(43,4)$ & $113(45,2)$ \\
Melanoderma & $19(61,3)$ & $12(38,7)$ & $31(12,4)$ \\
Sexo masculino & $88(62,9)$ & $52(37,1)$ & $140(56,0)$ \\
Sexo feminino & $71(64,6)$ & $39(35,5)$ & $110(44,0)$ \\
Hipertensão & $143(62,5)$ & $86(37,6)$ & $229(91,6)$ \\
HF + & $112(61,9)$ & $69(38,1)$ & $181(72,4)$ \\
DM2 & $61(68,5)$ & $28(31,4)$ & $89(35,6)$ \\
Dislipidemia & $90(57,7)$ & $66(42,3)$ & $156(62,4)$ \\
Total & $159(63,6)$ & $91(36,4)$ & $250(100)$
\end{tabular}

Legenda: ITB= índice tronozelo-braquial; $\mathrm{HF}+=$ positivo para doença cardiovascular; $\mathrm{DM} 2=$ diabetes mellitus tipo 2. ${ }^{*}$ Valores anormais: $\leq 0,9$ ou $>1,3$.

Fonte: Santana GQ, et. al., 2021.

Os fatores de risco inerentes ao paciente, ou seja, os fenômenos não modificáveis e próprios do indivíduo que o deixam mais susceptível aos eventos cardiovasculares, foram avaliados e os valores apresentados correspondem ao número e a porcentagem de ITB normais (entre 0,91 e 1,30) e anormais ( $\leq$ 0,9 ou $>1,3$ ) e o total de pacientes respectivamentede acordo com a condiçãopredisponente.

Tabela 2 - Fatores de risco cardiovasculares modificáveis associados ao ITB em pacientes submetidos a cateterismo de uma cidade de Minas Gerais em 2020.

\begin{tabular}{lccc}
\hline Fator de risco & ITB normal $\mathbf{n}(\%)$ & ITB anormal $\mathbf{n}(\%)$ & Total $\mathbf{n}(\%)$ \\
\hline Sedentarismo & $95(63,3)$ & $55(36,7)$ & $150(60,0)$ \\
Tabagismo & $39(68,4)$ & $18(31,6)$ & $57(22,8)$ \\
Ex-tabagista & $33(63,5)$ & $19(36,5)$ & $52(20,8)$ \\
Não tabagista & $87(61,7)$ & $54(38,3)$ & $141(56,4)$ \\
Etilismo & $37(78,7)$ & $10(21,3)$ & $47(18,8)$ \\
Ex-etilista & $18(62,1)$ & $11(37,9)$ & $29(11,6)$ \\
Não etilista & $104(59,8)$ & $70(40,2)$ & $174(69,6)$ \\
\hline
\end{tabular}

Legenda: ITB= índice tornozelo-braquial. *Valores anormais: $\leq 0,9$ ou $>1,3$.

Fonte: Santana GQ, et. al., 2021.

Já os fatores de risco modificáveis, ou seja, as condições que resultam do estilo de vida do indivíduo, que podem ser alteradas ao decorrer da vida e que, com mudanças, podem cursar com alteração da história clínica,deixando essas pessoas mais ou menos susceptíveis aos eventos tromboembólicos, foram avaliados e os valores apresentados correspondem ao número e a porcentagem de ITB normais (entre 0,91 e 1,30) e anormais $(\leq 0,9$ ou $>1,3)$ e o total de pacientes respectivamente de acordo com a condiçãopredisponente.

Idade, um fator de risco imutável, e IMC, um fator de risco variável, são valores numéricos, em que é calculado o valor médio da amostragem total, sendo encontrados $63,5 \pm 9,9$ anos e $25,8 \mathrm{Kg} / \mathrm{m}^{2}$, respectivamente, para cada fator investigado (Tabela 3 ). 
Tabela 3 - Fatores de risco cardiovascular associados ao ITB em pacientes submetidos a cateterismo em uma cidade de Minas Gerais em 2020

\begin{tabular}{lccc}
\hline Fator de risco & ITB normal média $\pm D P$ & ITB anormal $^{*}$ média $\pm D P$ & Total média $\pm D P$ \\
\hline Idade $($ anos $)$ & $62,55 \pm 10,37$ & $65,16 \pm 8,89$ & $63,50 \pm 9,92$ \\
IMC $\left(\mathrm{Kg} / \mathrm{m}^{2}\right)$ & $25,79 \pm 5,12$ & $25,91 \pm 4,80$ & $25,80 \pm 5,04$ \\
\hline
\end{tabular}

Legenda: ITB= índice tornozelo-braquial; IMC= índice de massa corporal; $\mathrm{DP}=$ desvio padrão. *Valores anormais: $\leq 0,9$ ou $>1,3$.

Fonte: Santana GQ, et. al., 2021.

Para cada item avaliado nas perguntas realizadas a partir do formulário, foi analisada a significância estatística $(p<0,05)$ que esses fatores apresentaram na sua relação com o ITB. Com isso, foi possível perceber que a etnia, o etilismo, a dislipidemia e a idade foram estatisticamente relevantes. Já as demais condições investigadas apresentaram $p>0,05$, embora muito prevalentes (Tabela 4).

Tabela 4 - Valor de p na relação do ITB com cada fator de risco investigado em pacientes submetidos a cateterismo cardíaco em uma cidade de Minas Gerais em 2020.

\begin{tabular}{lc}
\hline Fator de risco & $\mathbf{p}$ \\
\hline Etnia & $\mathbf{0 , 0 6 6}$ \\
Idade (anos) & $\mathbf{0 , 0 2 3}$ \\
Etilismo & $\mathbf{0 , 0 5 6}$ \\
Dislipidemia & $\mathbf{0 , 0 1 2}$ \\
Sexo & 0,783 \\
Sedentarismo & 0,915 \\
IMC $\left(\mathrm{Kg} / \mathrm{m}^{2}\right)$ & 0,549 \\
Hipertensão & 0,210 \\
Tabagismo & 0,673 \\
HF + & 0,360 \\
DM2 & 0,451
\end{tabular}

Legenda: ITB: índice tronozelo-braquial; HF+: positivo para doença cardiovascular; DM2: diabetes mellitus tipo 2; IMC: índice de massa corporal.

Fonte: Santana GQ, et. al., 2021.

\section{DISCUSSÃO}

Considerando a aterosclerose como um processo generalizado que pode envolver, não somente o território coronariano, mas, de forma isolada ou conjunta, os mais diversos territórios arteriais, é de extrema importância a avaliação integral do paciente, de modo a evitar as suasmanifestações e as suas complicações como a limitação da capacidade funcional, a piora de qualidade de vida e até mesmo o aumento da mortalidade (NEYELOFF JL, 2012; OLIVEIRA GHR, et al., 2011). A compreensão da formação de ateromatose vascular é de grande importância no intuito de combater precocemente a aterogênese e, dessa forma, reduzir a morbimortalidade por causas cardiovasculares (ATIK FA, et al., 2014; REIS SC., 2019).

A aterosclerose pode não causar sintomatologia clara por longos anos, entretanto, pode haver um processo de trombose como sua maior complicação, levando à oclusão do vaso. Pode manifestar-se em diversos sítios arteriais, como os territórios aórtico, carotídeo e coronário, e causar, dentre outras doenças, a DAOP, os acidentes vasculares encefálicos e a DAC. Assim, essa comorbidade possui grande influência nos altos índices de mortalidade cardiovascular (KULLO IJ e ROOKE TW, 2016; OLIN JW, et al., 2016; OLIVEIRA GHR, et al., 2011).

A obstrução arterial, seja do território que irriga os MMSS, os MMII ou as vísceras, evidencia a natureza sistêmica da doença aterosclerótica (OLIVEIRA GHR, et al., 2011). Dessa forma, a alteração do ITB é uma 
das primeiras manifestações da doença subclínica no processo de formação de placa aterosclerótica, principalmente naqueles pacientes com DAOP (FILHO JCJP, et al., 2015; NEYELOFF JL., 2012). Exemplo disso foi o estudo realizado na Cleveland Clinic que consistiu em uma análise de 1.000 pacientes consecutivos com diagnóstico prévio de DAOP, em que foi observado existência de lesão aterosclerótica, acometendo, pelo menos, uma artéria coronária epicárdica em mais de $90 \%$ dos pacientes e lesões tri arteriais graves em $28 \%$ dos casos (CHAGAS ACP, et al., 2003).

Um importante estudo epidemiológico realizado nos EUA, PAD Awareness, Risk and Treatment: New Resources for Survival (PARTNERS), que tinha como objetivo estabelecer a prevalência de DAOP na atenção primária, demonstrou que cerca de $50 \%$ dos pacientes desconheciam a presença dessa comorbidade, o que se mostrou como uma barreira para a atuação precoce e a prevenção secundária da isquemia cardiovascular (OLIVEIRA GHR, et al., 2011).

Sabe-se que a presença de DAOP é mais significativa com o avanço da idade devido a existência de outras doenças de base, apresentando, portanto, elevada prevalência na população de idosos (CRIQUI MH e ABOYANS V, 2015). Assim como no estudo de Framingham e no NHANES, que relatou associação da idade a partir dos 70 anos ou mais com o aumento da prevalência de DAOP, na análise da amostra deste estudo, o fator idade foi estatisticamente relevante $(p=0,02)$ no acometimento da doença, sendo então considerado um importante fator de risco cardiovascular (NETO SS e DO NASCIMENTO JLM, 2007).

Também foi encontrada significância estatística no presente trabalho, na relação de dislipidemia com a DAOP, achado esperado pela literatura, uma vez que maiores níveis de lípides sanguíneos favorecem a sua deposição em artérias, além da maior prevalência de ITB normal na população que não tinha DM2. Tais situações já eram previstas pelo estudo, de 500 pacientes, com indicação de cateterismo por suspeita de DAC, que comparou a prevalência dos fatores de risco nos grupos com obstrução periférica (279 pacientes) e sem esse bloqueio. O subgrupo com DAOP apresentou significância estatística com a presença de dislipidemia em $71 \%$ desses pacientes e em $61 \%$ dos pacientes sem a presença da doença. Em relação à diabetes, o grupo com aterosclerose periférica foi $3 x$ mais acometido, com $53 \%$ das pessoas e o subgrupo sem DAOP, com apenas 17\% (SUKHIJA R, et al., 2003).

Nesse contexto, os pacientes com Diabetes Mellitus possuem taxas de eventos cardiovasculares, em 10 anos, que podem atingir valores maiores que 30\% dependendo do risco estabelecido, sendo que, a presença de doença aterosclerótica subclínica, possível de ser diagnostica com ITB<0,9, é fator importante para definir o risco cardiovascular desses pacientes, já que essa patologia aumenta a chance da ocorrência de alterações relacionadas ao colesterol, cursando com maior probabilidade de um acometimento coronariano (FALUDI AA, et. al., 2017).

O consumo de álcool é sabidamente um fator de risco importante para diversas doenças sistêmicas, incluindo efeitos complexos na saúde cardiovascular. Apesar da magnitude desses eventos poder ser modulada pela genética e pelo ambiente, a quantidade e a frequência do consumo parecem interferir também. Pequenas a moderadas doses de álcool já podem ser suficientes para ativar mecanismos de homeostasia que geram processos inflamatórios e induzem a aterosclerose, além de alterações de circulação e stress oxidativo, gerando, até mesmo, alterações anatômicas nos vasos sanguíneos (PIANO $M R, 2017)$. Nesse contexto, a associação positiva do etilismo com DAOP já era esperada ( $p=0,026)$.

Principalmente entre os indivíduos mais jovens, é possível verificar alta chance de doença cardiovascular quando há um parente de primeiro grau diagnosticado com as patologias relacionadas ao coração. Apesarde tal fator de risco do estudo realizado não ter sido considerado significativo estatisticamente, entre o grupo com ITB anormal, a maior prevalência é de histórico familiar positivo $(75,8 \%)$ quando comparado com o negativo $(24,2 \%)$, revelando uma elevada associação desse fator de risco com a DAOP (WILSON PWF, 2020).

O exercício físico praticado de forma contínua e regular promove melhora em outros fatores de risco cardiovascular modificáveis, entre eles, tende a ajudar na tentativa de parar de fumar, melhora a sensibilidade a insulina e o controle glicêmico em pacientes com DM2 além de facilitar o controle de HAS e de dislipidemia. A escassez da prática de atividade física e o ganho de peso, além de serem fatores de risco 
para o desenvolvimento de fenômenos ateroscleróticos, agravam os outros fatores já pré-existentes (WILSON PWF, 2020). Nessa pesquisa, a maioria dos pacientes observados era sedentário, o que mostrou relevância estatística na alteração do ITB, além de ter sido observado um valor de média de IMC acima do ideal $\left(25,8 \mathrm{Kg} / \mathrm{m}^{2}\right)$, evidenciando a relação desse fator com o desenvolvimento de doenças coronarianas.

Na literatura não há fortes evidências sobre a influência do sexo na prevalência de DAOP na população em geral. Entretanto, é observado um aumento da mortalidade proveniente de comorbidades cardiovasculares nos homens, quando comparado às mulheres, que possuem chances reduzidas em, aproximadamente, $20 \%$ de evoluírem com tais complicações (WILSON PWF, 2020). Pode-se observar que, nos resultados encontrados, a maior prevalência de ITB alterado está no sexo masculino $(57,1 \%)$, enquanto as mulheres representam $42,8 \%$ desse grupo.

Entre os pacientes com doenças cardiovasculares, a HAS é um fator de risco já bem estabelecido pela literatura (ALVIM RO, et al., 2018; WILSON PWF, 2020). Apesar de o fator hipertensão não ter sido estatisticamente significativo na amostra de ITB alterado, o número de pacientes portadores de HAS (229) foi bem maior que o número de indivíduos saudáveis (21), o que se mostra como um número importante, uma vez que todos os pacientes que foram avaliados possuíam elevado risco cardiovascular devido ao acometimento coronariano.

O tabagismo tem forte relação com certas manifestações cardiovasculares, como a claudicação intermitente e a isquemia do miocárdio. O cigarro eleva em, aproximadamente, quatro vezes o risco para 0 aparecimento de um IAM e estimula em torno de uma década o surgimento da claudicação intermitente. Através de estudos, a relação dos fumantes com a probabilidade de desenvolverem um IAM é seis vezes maior em mulheres e três vezes maior nos homens, transparecendo a forte relação do cigarro com o mau prognóstico de doenças cardíacas em ambos os sexos. Além disso, há uma quantidade maior de homens que fumam em relação ao sexo feminino, podendo estar relacionado ao sexo masculino ter maior prevalência de eventos cardiovasculares e gerar pior prognóstico (ALVIM RO, et al., 2018; WILSON PWF, 2020).

\section{CONCLUSÃO}

Os valores estatisticamente significativos encontrados da associação dos fatores de risco cardiovascular com DAOP em pacientes submetidos a cateterismo cardíaco, demonstram a eficácia do cálculo de ITB e a necessidade de inclusão desse índice na prática clínica como um preditor de risco importante para doença coronariana e, portanto, auxiliar nas medidas terapêuticas a serem tomadas a partir do momento da confirmação diagnóstica.

\section{AGRADECIMENTOS}

Os autores do presente estudo agradecem a Faculdade de Medicina de Barbacena - Fundação José Bonifácio Lafayette de Andrada que possibilitou a realização da pesquisa e por todo o apoio fornecido e principalmente a todos os pacientes que se prontificaram a responder o questionário aplicado e permitiram que o exame clínico fosse realizado.

\section{REFERÊNCIAS}

1. ALVIM RO, et al. Prevalence of Peripheral Artery Disease and Associated Risk Factors in a Brazilian Rural Population: The Baependi Heart Study. International Journal of Cardiovascular Sciences [online], 2018; 31(4): 405413.

2. ANKLE BRACHIAL INDEX COLLABORATION, et al. Ankle brachial index combined with Framingham risk score to predict cardiovascular events and mortality: a meta-analysis. JAMA, 2008; 300(2): 197-208.

3. ARAÚJO ALGS, et al. Frequência e fatores relacionados ao índice tornozelo-braquial aberrante em diabéticos. Jornal Vascular Brasileiro [online], 2016, 15(3): 176-181.

4. ATIK FA, et al. Fatores de risco de ateromatose da aorta em cirurgia cardiovascular. Revista Brasileira de Cirurgia Cardiovascular [online], 2014; 24(4): 487-493.

5. CHAGAS ACP, et al. Doença aterosclerótica não coronariana. 1a ed. São Paulo: BBS Editora; 2003; 254p. 
6. CRIQUI MH, ABOYANS V. Epidemiology of peripheral artery disease. Circulation research, 2015; 116(9): $1509-1526$.

7. FALUDI AA, et al. Atualização da diretriz brasileira de dislipidemias e prevenção da aterosclerose. Arquivos Brasileiros de Cardiologia, 2017; 109(2): 1-76.

8. FILHO JCJP, et al. O papel do índice tornozelo-braço no prognóstico cardiovascular e na predição de lesões de órgãos-alvo em pacientes hipertensos. Revista Brasileira de Hipertensão, 2015; 22(4): 119-125.

9. HIRSCH AT, et al. Peripheral Arterial Disease Detection, Awareness, and Treatment in Primary Care. JAMA, 2001; 286(11): 1317-1324.

10. KULLO IJ, ROOKE TW. Clinical Practice. Peripheral artery disease. The New England journal of medicine, 2016; 374(9): 861-871.

11. LIU L, et al. Prognostic Value of Abnormal Ankle-Brachial Index in Patients With Coronary Artery Disease: A MetaAnalysis. Angiology, 2020; 71(6): 491-497.

12. NETO SS, DO NASCIMENTO JLM. Doença arterial obstrutiva periférica: novas causas de risco. Revista Paraense de Medicina, 2007; 21(2): 35-39.

13. NEYELOFF JL. Rastreio de doença arterial obstrutiva periférica: impacto no tratamento farmacológico de pacientes hipertensos e análise de custo-efetividade para reestratificação de risco cardiovascular. Tese (doutorado) Universidade Federal do Rio Grande do Sul, Faculdade de Medicina, Programa Pós-graduação em Ciências da Saúde: Cardiologia e Ciências Cardiovasculares, Porto Alegre, 2012, $127 f$.

14. NUNES FGF, et al. Índice tornozelo-braquial em pacientes de alto risco cardiovascular. Revista Brasileira de Cardiologia, 2012; 25(2): 94-101.

15. OLIN JW, et al. Peripheral artery disease: evolving role of exercise, medical therapy, and endovascular options. Journal of the American College of Cardiology, 2016; 67(11): 1338-1357.

16. OLIVEIRA GHR, et al. Doença ateromatosa multivascular: relato de caso. Revista Médica (São Paulo), 2011; 90(3): 144-148.

17. PETRACCO AM, et al. Assessment of the Relationship of Ankle-Brachial Index With Coronary Artery Disease Severity.International Journal of Cardiovascular Sciences [online], 2018; 31(1): 47-55.

18. PIANO MR. Alcohol's Effects on the Cardiovascular System. Piano, Mariann R. "Alcohol's Effects on the Cardiovascular System." Pesquisa sobre álcool: revisões atuais, 2017; 38(2): 219-241.

19. PRESTI C, et al. Doença arterial periférica obstrutiva de membros inferiores diagnóstico e tratamento. Projeto de Diretrizes da Sociedade Brasileira de Angiologia e Cirurgia Vascular, 2015, 33f.

20. REIS SC. Uma revisão bibliográfica sobre aterosclerose. Revista Eletrônica Acervo Científico, 2019; 2 : e444.

21. SABEDOTTI M, et al. Índice Tornozelo-Braquial como Preditor de Doença Coronariana Significativa em Pacientes Submetidos à Angiografia Coronária. Revista Brasileira de Cardiologia Invasiva [online], 2014; 22(4): 359-363.

22. SOCIEDADE BRASILEIRA DE CARDIOLOGIA. Atualização da Diretriz de Prevenção Cardiovascular da Sociedade Brasileira de Cardiologia. Arquivos Brasileiros de Cardiologia, 2019; 113(4): 787-891.

23. SUKHIJA R, et al. Prevalence of left main coronary artery disease, of three- or four-vessel coronary artery disease, and of obstructive coronary artery disease in patients with and without peripheral arterial disease undergoing coronary angiography for suspected coronary artery disease. American journal of cardiology, 2003; 92(3): 304-305.

24. WILSON PWF. Overview of established risk factors for cardiovascular disease. Uptodate, Última atualização em: 4 maio 2020. 